\title{
GUEST EDITORIAL: \\ SPECIAL ISSUE ON ADVANCED INFORMATION SYSTEMS ENGINEERING
}

\author{
SjaAK BrinkKemper ${ }^{1}$, Anthony I. WASSERmaN ${ }^{2}$ and Gerard Wijers ${ }^{3}$ \\ ${ }^{1}$ Centre for Telematics and Information Technology, University of Twente, P.O. Box 217, 7500 AE Enschede \\ The Netherlands \\ ${ }^{2}$ Interactive Development Environments, Inc., 595 Market Street, 10th Floor, San Francisco, CA 94105, USA \\ ${ }^{3}$ ID Research, Kastanjelaan 4, 3833 AN Leusden, The Netherlands
}

There is a huge investment in existing software systems, with organizations spending huge sums of money on the maintenance of old systems. As organizations transform the way that they do business, they must also transform their legacy systems to take advantage of these fundamental changes in technology. Lowering costs by moving applications to modern platforms and development approaches is a critical need for most organizations.

The need for modernization is driven by fundamental changes in the computer industry, including the movement from proprietary systems to open systems, the movement of applications from mainframes to client-server environments, and the resulting change from alphanumeric to graphical user interfaces. The transition to open client-server computing environments is occurring very rapidly, and has led to widespread adoption of standards for cross-platform networking and database access.

Modernization of application development often involves use of a different development process, as well as a new set of tools. This special issue presents a selected number of advancements in Information Systems Engineering presented at the CAISE '94 Conference, held in Utrecht, the Netherlands in June 1994. CAISE '94 was the sixth International Conference on Advanced Information Systems Engineering, continuing the tradition of the series of a major platform for researchers and practitioners from acadcmia and industry to discuss and exchange new developments in methods, techniques and tools to realize advanced information systems.

This special issue contains revised and expanded versions of five of the highest ranked papers selected by the Program Committee from the more than 130 submissions. The first three papers describe innovations of IS engineering techniques and tools. Jackson, Embley, and Woodfield describe tool support for an object-oriented systems analysis method based on a formal model. Their object-oriented specification model, called OSS, is supported by a tool (IPOST) that allows generation of executable prototypes. With today's multitude of object-oriented methods, this formal approach contributes to the deep understanding of the object-oriented paradigm and its relationship to the generation of application systems. To integrate the information representation and the organizational communication, Johannesson proposes the application of speech-act theory. Speech acts are short communicative acts between two roles in terms of an established conceptual schema. The paper introduces a formal specification technique, discourse structures, composed of natural units of speech acts to model organizational communications in a systematic way. The problem of schema integration based on behavioural information is addressed in the paper by Thieme and Siebes. The standard way of schema integration is complemented with steps to take the syntactic and semantic properties of the operations (methods) into account. The remaining two papers deal with the support of the system engineering process. Conradi, Hagaseth, and Liu address the planning of complex transactions (e.g. updates of documents) in a software engineering environment supporting concurrent work. Their EPOS Software Process Environment is extended with a transaction planning assistant, called TRAPLAS, that assists in resolving conflicts when several engineers are accessing the same project repository. Support for guiding the systems development process is presented by Rolland, Souveyet, and Moreno. As the context of a 
development context is important to decision making, they introduce a decision-oriented software process modelling formalism with three types of contexts: executive-based context, choice-based context, and plan-based context. Their approach is illustrated by a case study with an Air Traffic Control system.

We believe that these selected papers from CAISE '94 each make a significant contribution to the field of Information Systems Engineering. We hope that they can serve as valuable sources for future research work and practical application.

Finally, we wish to thank all people involved in the production process for this special issue, especially the members of the CAISE ' 94 program committee, the authors, and all the reviewers.

Sjaak Brinkkemper Anthony I. Wasserman

Gerard Wijers March 1995 